\title{
Prognosen beim Glioblastom: zu pessimistisch?
}

\begin{abstract}
„Wie lange habe ich noch?" Diese drängendste aller Fragen jedes Krebspatienten lässt sich in Zukunft eventuell besser und detaillierter beantworten. „Bedingte Wahrscheinlichkeiten“ erlauben es, die verbleibenden Jahre nicht nur zum Zeitpunkt der Diagnose, sondern nach einem, zwei oder drei überstandenen Jahren abzuschätzen. Bei GlioblastomPatienten fanden US-Forscher damit einen Effekt, der optimistisch stimmt.
\end{abstract}

$\mathrm{N}$ icht nur nach der Krebsdiagnose, sondern auch wenn er mit einer Therapie bereits eine gewisse Zeit überlebt hat, möchte der Patient wissen, wie die Chancen auf ein längerfristiges Überleben stehen. Hilfreich in diesem Zusammenhang scheint die bedingte Wahrscheinlichkeit (conditional probability), fand ein Team vom National Cancer Institute in Rockville, Maryland, an 498 GlioblastomPatienten heraus. Herkömmlichen Schätzungen auf der Basis von Kaplan-MeierKurven zufolge überleben Patienten unter einer Kombination aus Bestrahlung und Temozolomid (TMZ) mit einer 27\%igen Wahrscheinlichkeit zwei Jahre.

Mithilfe der bedingten Wahrscheinlichkeit ließ sich in der Studie bei Patienten, die bereits ein, zwei, drei oder vier Jahre überlebt hatten, die Chance auf ein Überleben um ein weiteres Jahr auf 35\%, 49\%, $69 \%$ bzw. $93 \%$ beziffern. Sechs Monate nach Diagnose hatte die bedingte Wahrscheinlichkeit noch $72 \%$ betragen. Dies galt sowohl für Patienten, die zusätzlich zur Bestrahlung Temozolomid und entweder Thalidomid (TTRT) oder cis-Retinolsäure (RTRT) erhalten hatten, als auch für Patienten unter einem älteren Regime ohne Temozolomid (Pre-TMZ).

Die Überlebenschancen unter einer Therapie mit dem erst kürzlich eingeführten Regime Temozolomid plus Erlotinib (OTRT) waren überwiegend besser und wurden daher separat betrachtet. Hier lagen die bedingten Überlebenschancen bei $48 \%$, $71 \%$, $71 \%$ und $69 \%$. Die nach Kaplan-Meier ermittelten Überlebensraten betrugen für die TTRT-, RTRT und PreTMZ-Patienten nach sechs Monaten, einem, zwei, drei und vier Jahren $80 \%$, $58 \%, 20 \%, 10 \%$ und $7 \%$ bzw. unter OTRT $92 \%, 68 \%, 32 \%, 23 \%$ und $16 \%$.

Verglichen mit der nicht bedingten Wahrscheinlichkeit, ein Jahr nach Diagnose zu überleben (58\%), war die bedingte Überlebenswahrscheinlichkeit

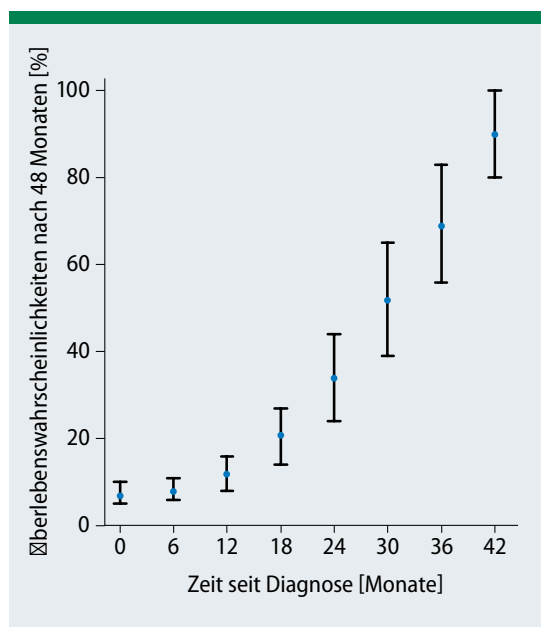

Die bedingte Wahrscheinlichkeit, das Glioblastom zu überleben, steigt nach vier Jahren Therapie mit Pre-Temozolomid-Regimes oder Temozolomid plus Thalidomid bzw. cis-Retinolsäure.

zunächst rapide gesunken, um nach eineinhalb Jahren wieder anzusteigen. Nach drei Jahren hatte die bedingte 1-JahresWahrscheinlichkeit mit $69 \%$ die auf Basis der Kaplan-Meier-Kurve geschätzte Überlebensrate bereits deutlich überholt. Damit ist die Prognose für einen Patienten, der bereits drei Jahre überlebt hat, wieder so gut wie kurz nach Diagnose. Trotz des rapiden Abfalls der Überlebenswahrscheinlichkeit in den ersten zwei Jahren kann man davon ausgehen, dass sich die Prognose bei Patienten, die zwei Jahre überleben, wieder verbessert.

Fazit: Die bedingte Wahrscheinlichkeit konnte in der vorliegenden Studie die Überlebenszeit von Patienten mit Glioblastoma multiforme verlässlicher - und optimistischer - abschätzen als die Kaplan-Meier-Kurve. Elke Oberhofer

Polley MYC et al. Conditional probability of survival in patients with newly diagnosed glioblastoma. J Clin Oncol. 2011;29:4175-80. 